\title{
The New Challenges of the Albanian Press and State Education on Early Twentieth Century
}

\author{
Esmeralda Dani \\ University "LuigjGurakuqi", Faculty of Education, Department of Teaching, Shkodër, Albania \\ Email: esmeraldahoti@yahoo.it \\ Eranda Bilali \\ University “LuigjGurakuqi”, Faculty of Education, Department of Teaching, Shkodër, Albania \\ Email: erandabilali@yahoo.com
}

\section{Doi:10.5901/ajis.2016.v5n3s1p378}

\section{Abstract}

\begin{abstract}
After the fall of the communist regime in Albania it has opened a great debate on the so-called "re-writing of history", a complex process of deconstruction of the scientific methods of the regime and of the myths that have impregnated the Albanian historiography, that actually impede young Albanians to identify with their own historical and cultural roots, so important in facing the challenges of globalization identity. The purpose of this article is to identify the challenges of the press and of the Albanian Government, in 1912, to achieve the education of the masses. The main objective is to understand whether the Albanian press has stressed the importance of national identity formation and education of the masses, and also the importance of the legislative organization of national education in the early twentieth century. To achieve this purpose has been used desk research method. After the analysis of original documents and articles of the Albanian national archive, we can argue that 1. the Albanian state education story began after the publication in newspapers of three documents: Luigj Gurakuqis' announcement of September 6, the circular of September 12 and "Article 29" civil administration law of 22 November $1913 ; 2$. The Albanian press actually has propagandize the love for the country and for education, helping the government in achieving its main objective of state education.
\end{abstract}

Keywords: Albanian legal system, mass education, patriotic education, state education, early twentieth century

\section{Introduzione}

Recentemente, negli ambienti intellettuali albanesi, dopo la caduta del regime comunista, si è aperto un grande dibattito sulla cosiddetta "ri-scrittura della storia", un processo complesso di decostruzione dei metodi scientifici del regime e delle mitologie che hanno impregnato la storiografia. Tali necessità di presentare una storiografia diversa hanno ovviamente suscitato diverse polemiche ${ }^{1}$, in parte perché l'apparato interpretativo e concettuale ereditato dal regime è ancora dominante, in parte perché la storiografia albanese abbandona a fatica la presunta missione patriottica, per la quale il passato deve essere interpretato in chiave funzionale, ossia in funzione alla nazione e che rende spesso la storiografia albanese particolare per l'uso politico della storia che continua a fare. Per questa ragione bisogna ri-studiare tutti i documenti originali in un'altra ottica molto più razionale e basata sul desiderio della verità. Una verità che aiuta molti giovani a capire le dinamiche della costruzione delle proprie identità, sia personale che nazionale. In effetti, una delle sfide maggiore del globalismo e di trovare delle forme di educazione per conservare le identità nazionali per non perdersi nell'immensa identità globale.

\section{Metodologia}

Per raggiungere gli obiettivi della ricerca è stato usato la metodologia qualitativa, il metodo desk resarch, attraverso il quale è stato possibili analizzare i documenti originali del periodo interessato.

${ }_{1}^{1}$ Fuga A., Vdekja e historisë së letërsisë, "Lemba", 12 (2008), Valaj, Shkodër, p. 5. 


\section{Discussione}

Proporre progetti educativi e programmi sull'istruzione agli esordi dello stato albanse, in realtà, non erano più sufficienti alla fragile situazione educativa albanese, perciò, assieme alla proclamazione dell'Indipendenza emergeva il bisogno dell'organizzazione legislativa dell'istruzione pedagogica nazionale. Sul territorio del nuovo Stato albanese, nel 1912, funzionavano una scuola normale solo per ragazzi e poche scuole elementari prive di carattere nazionale. Pertanto, il programma d'insegnamento del governo di Valona doveva comprendere i seguenti orientamenti principali: definire la lingua albanese come lingua ufficiale dello Stato e adoperarla nell'amministrazione albanese, nelle scuole e nell'esercito; fondare le basi legislative dell'istruzione nazionale; tentare di costruire un sistema istruttivo nazionale per tutti; preparare insegnanti nelle scuole normali, nei corsi accelerati e attuare nuove opportunità per gli studenti migliori nel proseguire gli studi nelle università migliori².

Se educare è un'azione politica, le forme che tale azione assume, variano a secondo del contesto nel quale l'educatore si trova ad operare, e già Rousseau sosteneva che «dove esiste la patria occorre educare i cittadini, [e] dove la patria non esiste non è possibile educare cittadini, visto che neppure essi possono esistere. In tale condizione è quindi necessario ripiegare su una soluzione diversa, educando l'uomo. Ogni altra soluzione intermedia è destinata a fallire, poiché pone l'essere umano in continua contraddizione con se stesso, perennemente esitante tra inclinazione e dovere». La soluzione educativa, alla fine, rimane sempre la formazione dell'uomo, una formazione che cercherà di creare le condizioni ideali per il giovane che vuole contribuire per la propria comunità d'appartenenza ${ }^{3}$.

II Ministero dell'Istruzione del Governo Provvisorio di Valona, dopo aver occupato gli edifici della prima scuola albanese di Valona, la Muradie, diede enorme importanza alla diffusione immediata dell'istruzione elementare e dell'educazione, poiché strumento indispensabile nella formazione dei cittadini ${ }^{4}$, da un annuncio del giornale La rinascita Albanese (Përlindja Shqiptare) ${ }^{5}$, organo di stampa ufficiale del governo ${ }^{6}$, dall'apertura di due scuole "Normali Preparative", una basata sulla riapertura della normale di Elbasan chiusa nel $1910^{7}$ e l'altra a Berat, indirizzate alla formazione accelerata dei maestri elementari8.

L'annuncio ufficiale del Ministero d'Istruzione affermava che:

Il governo albanese ha deciso di aprire in ogni Prefettura una scuola Normale Preparatoria, nella quale possono partecipare tutti gli alunni che hanno finito la ruzhdije o qualsiasi altra scuola equivalente, tutti gli albanesi che hanno lavorato come insegnanti, chi non soffre di malattie contagiose, quelli che non hanno compiuto 30 anni e chi risulta vincitore del concorso d'ammissione'.

In seguito, il responsabile del Ministero d'Istruzione dichiarava che ormai «ai patrioti spetta di servire alla nazione con professionalità e umiltà. A tutti i maestri dell'insegnamento, a quelli che ardiscono dire che sono i messaggeri della nuova Albania, che si facciano avanti per far rinascere le speranze dei giovani. [...] Chi non vuole un Albania orfana deve lottare contro l'ignoranza. E viva i fausti che diventeranno gli insegnanti della libera Arbënia!» ${ }^{10}$.

Con il termine "preparativi" s'intendevano «le classi propedeutiche alle normali e che includevano un contingente eterogeneo di alunni, sia in base alle conoscenze godute sia in base alla formazione generale culturale. Erano usate delle forme particolari d'insegnamento per rendere abili tutti gli alunni [...] i quali o esercitavano professioni diverse 0 non avevano ancora finito i loro studi [...] Principalmente si attuavano materie di carattere pedagogiche, racchiudendo anche problemi d'organizzazione della nuova scuola albanese» ${ }^{11}$. Le poche possibilità economiche e i poveri mezzi organizzativi rendevano impossibile la rinnovazione delle infrastrutture educative, ma «questo piano ambizioso fu aiutato dall'apertura delle scuole preparativo di Berat, che nell'anno scolastico 1913-1914 ebbe un direttore, 3 insegnanti a

\footnotetext{
2 Shllaku, Shkollat klerikale, cit., pp. 94-95.

${ }^{3}$ Nardi E., Oltre l'Emilio. Scritti di Rousseau sull'educazione, Franco Angeli, Milano, 2005, p. 243.

${ }^{4}$ Ibid., p. 246.

${ }^{5}$ S. Gj. D., Ministria e Arsimit, "Përlindja Shqiptare", 1(1913), Vlorë, p. 2.

${ }^{6}$ Kashari Q., Qeveria e përkohshme e Vlorës për arsimin dhe shkollën kombëtare shqiptare, "Revista Pedagogjike", 4 (1982), Tiranë, p. 110.

7 Osmani, Reflekse etnopedagogjike, cit., p. 261.

${ }^{8}$ Ibid., pp. 267-268.

9 Ministria e Arsmit, "Përlindja shqiptare", cit., p. 2.

10 Ibidem.

11 Ibid., p. 267.
} 
tempo pieno e 2 a chiamata, e di Elbasan»12. Entro il primo anno della sua attività, queste scuole riuscirono a preparare il primo contingente d'insegnanti in solo quattro mesi, prendendo maggiormente la fisionomia di un corso professionale. Come testi d'insegnamento venivano usati la Grammatica di Sotir Peci, l'Aritmetica di Kristo Dako, Mirëvetija di Jani Vretos, Rreze drite di Asdren, le opere di Naim Frashëri. Le materie scientifiche insegnate includevano lingua, aritmetica, pedagogia e psicologia, mentre quelle professionali includevano appunti tradotti dai testi stranieri ${ }^{13}$.

La questione dell'educazione di massa attraverso l'istruzione era considerata l'unico modo per rigenerare la fragile nazione nascente:

Non esiste altro ambito più arretrato di quello d'istruzione in Albania! II governo non dimostra segni concreti di ripresa. [...] Anche per questo anno scolastico le scuole elementari saranno aperte in tutte le città, campagne e province; un scuola preparativa verrà aperta a Elbasan, e scuole di più alto livello siamo pronti ad aprirle se i sviluppi lo consentiranno. [...] Spesso abbiamo pubblicato degli annunci riguardando l'ingaggio di nuovi insegnanti, ma poco hanno risposto. [...] I giovani devono sapere che non esiste professione più sacra di quella dell'insegnante ${ }^{14}$.

Durante tutto questo tempo, di serio impegno e di lotte continue per coltivare il sapere e la consapevolezza della propria esistenza, gli intellettuali del movimento nazionale non riuscirono a fondere vere e proprie riviste di carattere istruttivo-pedagogico, ma, nella stampa del periodo, avevano pubblicato soltanto degli articoli sporadici con elementi educativi, come l'articolo scritto da Emin Matraxhiu, il 28 agosto 1913, su come il giovane albanese doveva scoprire dentro di sé la forza di confrontarsi con la propria disperazione e pigrizia, per raggiungere la speranza:

Gioventù! II tempo sta per finire, perciò è responsabilità individuale di impegnarsi nello sviluppo, nel miglioramento e nella salvezza di questa sciagurata nazione [...] Gioventù! Trova una soluzione alla pigrizia, quella che nuoce, e buttatela via [...] Voltare le spalle alla patria è una vigliaccheria, un'astuzia; la disperazione, ancora di più, è un grande tradimento ${ }^{15}$.

La scuola e la famiglia, in questo collettivo impegno educativo, dovevano dedicare del tempo qualitativo all'educazione mentale e fisica del bambino, come d'altronde già nel 1888 Edmondo de Amicis asseriva con il celeberrimo Cuore ${ }^{16}$. Per raggiungere la consapevolezza dei propri bisogni emozionali e identitari non occorreva raccontare inutili e false verità sul mondo e sui rapporti umani17. Come sosteneva anche Luis Rollin:

É necessario insegnare agli allievi di trasformare in abitudine la verità e non arrestare le loro azioni dinamiche. Di conseguenza, dobbiamo permetterli di comportarsi con naturalezza per fare essi stessi la differenza. [...] E, in questo modo, il giudizio, lo scopo implicito dell'andamento scolastico, diventerà l'oggetto chiaro dell'attività didattica. Quest'attività avrà successo solo se l'allievo riuscirà nella sua vita a incontrare il bon gusto con il sentimento della verità ${ }^{18}$.

Uno dei giornalisti più importanti che espresse apertamente le sue opinioni sulle politiche d'istruzione del governo di Valona fu Mihal Grameno, già citato nei capitoli precedenti, che quando scrisse sul progresso dell'istruzione in Albania dimostrò tutto il suo amore per la patria e per la libertà verso la conoscenza:

Dall'istruzione che riceveranno i giovani, fiorirà la civilizzazione dell'Albania e la strada verso le nazioni europee. Naturalmente, l'albanese è intelligente ma questo non basta, poiché senza istruzione non possiamo mai raggiungere una formazione elevata, giacché la scuola lo fa diventare un patriota e un buon cittadino. [...] La responsabilità è enorme, [...] e ci vuole coraggio ad affrontare tutte le difficoltà, anche la mancanza degli insegnanti qualificati. Ciononostante, il ministro decise di aprire delle scuole normali preparatorie, dalle quali, dopo 4 mesi d'insegnamento, verranno distribuiti insegnanti in tutto il paese. [...] Mentre concernenti le scuole speciali, il signor ministro, ha pubblicato una circolare in cui dimostra tutti i passaggi da seguire ${ }^{19}$.

La circolare pubblicata nel giornale ufficiale del Ministero d'Istruzione, comunicava che tutte le scuole elementari sarebbero state aperte il 16 settembre e tutti gli alunni saranno stati obbligati a presentarsi nelle prime lezioni, mentre le

\footnotetext{
12 Koci K., Arsimi në periudhën e qeverisë së përkohshme të Vlorës 1912-1914, "Revista Pedagogjike", 1 (1978), Tiranë, pp. $95-116$.

13 Osmani, Reflekse etnopedagogjike, cit., pp. 269-270.

14 Gurakuqi L., Ministria e Arsmit, "Përlindja Shqiptare", 9 (1913), Vlorë, p. 5.

${ }_{15}$ Matraxhiu E., Dëshpërimi, Përtimi, "Përlindja Shqiptare", 9 (1913), Vlorë, p. 2.

${ }^{16}$ Russo A., Ferrari M., Morandi M. (a cura di), Patrioti si diventa. Luoghi e linguaggi di pedagogia patriottica nell'Italia unita, Franco Angeli, Milano, 2009, p. 28.

17 M.A.SH., Historia e arsimit, cit., pp. 280-281.

18 Mesnard P., Rollin farkëton mendimin për arsimin e mesëm, in Chateau J. (a cura di), Pedagogët e mëdhenj, Toena, Tiranë, $2000, p$. 176, 177.

19 Grameno M., Arsimi në Shqipëri, "Përlindja shqiptare”, 11(1913), Vlorë, pp. 2-3.
} 
scuole speciali dovevano obbligatoriamente adempiere le seguenti condizioni per esercitarsi liberamente:

1. Conseguire il permesso dal Governo; 2. La lingua ufficiale sarà la lingua albanese; 3. Avere i programmi, i libri e i diplomi approvati dal Ministero dell'Istruzione; 4. Rispettare le politiche di ogni Comune dello Stato. Nessuna scuola potrà cominciare l'anno scolastico se i direttori non rispetteranno gli obblighi annunciati20.

Per riorganizzare la vita politica, sociale, economica e culturale, la condizione minima, indubbiamente, era il raggiungimento della libertà, un requisito importante ma anche impegnatissimo, visto la poca esperienza del nuovo governo, il quale s'imbarcò subito in grandi difficoltà di organizzazione interna. L'attività dei governanti era indirizzata maggiormente alla costruzione della base legislativa dell'istruzione, in primis l'inserimento in tutte le istituzioni statali della lingua albanese come lingua ufficiale. Così, La legge adattata dall'Amministrazione civile dell'Albania pubblicato il 22 novembre del 1913 nel giornale ufficiale, edificava la prima legislazione istruttiva21, ove si specificava che ogni Prefettura doveva creare una Direzione dell'Istruzione e il direttore di questa Direzione aveva il dovere di osservare la costruzione e l'allestimento dei locali scolastici, l'esame d'ammissione degli insegnanti, il sistema migliore dell'apprendimento, incoraggiare lo sviluppo delle Belli Arti e aiutare i maestri in difficoltà22. Per di più, il direttore dell'istruzione era anche il Presidente del Consiglio d'Istruzione di Prefettura, il quale doveva eseguire tutti quegli ordini e decisioni che assicuravano lo sviluppo dell'istruzione nazionale, altresì quelli che provenivano dal Ministero dell'Istruzione ${ }^{23}$.

In sintesi, possiamo sostenere che la storia dell'istruzione statale albanese ebbe inizio dopo la pubblicazione di tre documenti: l'annuncio del 6 settembre di Luigj Gurakuqi, la circolare del 12 settembre ${ }^{24}$ e l'articolo 29 della legge sull'Amministrazione civile del 22 novembre $1913^{25}$.

L'impegno era serio, tenace e complessivo in quella che sia il governo sia gli intellettuali albanesi consideravano l'emergenza del momento: formare insegnati degni dell'educazione patriotica. Effettivamente, l'albanismo concepito agli esordi dai cattolici di rito bizantino situati in Italia e dagli ortodossi dei Balcani, era in una certa maniera connessa alla religione. I nazionalisti albanesi dovevano difendere l'idea che la propria nazione, anche se di maggioranza musulmana, era degna di considerarsi europea e di fondare il proprio Stato ${ }^{26}$.

\footnotetext{
20 Ministria e Arsimit, "Përlindja shqiptare", 12 (1913), Vlorë, p. 6.

${ }^{21}$ Kashari, Qeveria e përkohshme e Vlorës, cit., p. 110.

22 Ministria e Arsimit, Kanun i përshtatshëm i Aministratës qivile të Shqipënisë, "Përlindja e Shqipnisë”, 28 (1913), Vlorë, p. 3.

23 Ibidem.

24 Jahaj, Kur çelin shkronjat, cit., p. 98.

${ }^{25}$ Ministria e Arsimit, Kanun i përshtatshëm i Aministratës qivile të Shqipënisë, cit., p. 3.

${ }^{26}$ Clayer, Në fillimet e nacionalizmit shqiptar, cit., pp. 640-641.
} 